\title{
Analysis of Dynamic Characteristics for Wide Slider Bearings with an Exponential Film Profile
}

\author{
Jaw-Ren Lin \\ Professor, Department of Mechanical Engineering, Nanya Institute of Technology, P.O. Box 324-22-59, Chung-Li, \\ Taiwan, R.O.C., jrlin@nanya.edu.tw \\ Chi-Ren Hung \\ Instructor, Department of Mechanical Engineering, Nanya Institute of Technology, P.O. Box 324-22-59, Chung-Li, \\ Taiwan, R.O.C.
}

Follow this and additional works at: https://jmstt.ntou.edu.tw/journal

Part of the Mechanical Engineering Commons

\section{Recommended Citation}

Lin, Jaw-Ren and Hung, Chi-Ren (2004) "Analysis of Dynamic Characteristics for Wide Slider Bearings with an Exponential Film Profile," Journal of Marine Science and Technology. Vol. 12: Iss. 3, Article 11.

DOI: $10.51400 / 2709-6998.2243$

Available at: https://jmstt.ntou.edu.tw/journal/vol12/iss3/11

This Research Article is brought to you for free and open access by Journal of Marine Science and Technology. It has been accepted for inclusion in Journal of Marine Science and Technology by an authorized editor of Journal of Marine Science and Technology. 


\title{
Short Paper
}

\section{ANALYSIS OF DYNAMIC CHARACTERISTICS FOR WIDE SLIDER BEARINGS WITH AN EXPONENTIAL FILM PROFILE}

\author{
Jaw-Ren Lin* and Chi-Ren Hung**
}

Key words: dynamic characteristics, bearings, sliders, exponential film shape.

\begin{abstract}
Analysis of dynamic characteristics plays an important role in designing hydrodynamic bearings. Based upon the thin-film lubrication theory, the analysis of dynamic characteristics for a wide slider with exponential film profiles is presented when taking into account the bearing squeezing action. By applying a small perturbation technique to the dynamic Reynolds-type equation, both of the steadystate performance and the dynamic characteristics are then evaluated. Comparing with those of slider bearings with an inclined-plane film shape, higher values of the load-carrying capacity, stiffness coefficient and damping coefficient are predicted for the bearing with larger values of the inlet-outlet film ratio. These results provide engineers useful information to design machine elements and bearing systems.
\end{abstract}

\section{INTRODUCTION}

Slider bearings are designed for supporting transverse load in engineering practice. An understanding of the steady-state performance and dynamic characteristics of bearings taking into account the geometry and different operating conditions is important. By considering different film shapes, bearing characteristics have been analyzed [4, 8]. Advanced studies are presented by considering different operation conditions, such as the viscosity variation across the film [9], the inertia force effects $[6,10,13]$, the turbulent flows [5, 15], the temperature variation of fluid film [8], the thermal effects $[1,11,12,14]$. All the above studies, however, focus upon the performance of slider bearing operating

Paper Submitted 12/25/03, Accepted 05/31/04. Author for Correspondence: Jaw-Ren Lin.E-mail: jrlin@nanya.edu.tw.

*Professor, Department of Mechanical Engineering, Nanya Institute of Technology, P.O. Box 324-22-59, Chung-Li, Taiwan, R.O.C.

**Instructor, Department of Mechanical Engineering, Nanya Institute of Technology, P.O. Box 324-22-59, Chung-Li, Taiwan, R.O.C. under the steady-state situation, in which the effects of dynamic squeezing motion are neglected. It is well known that the steady-state performance provides a basic reference in designing bearings. In order for avoiding runner-pad contact and for predicting stability behavior of the bearing, a study of dynamic characteristics shows more important. Since slider-bearing surfaces operate mainly upon the wedge-action principle, an understanding of the dynamic stiffness and damping behaviors is helpful in designing the bearing. In a previous study, Lin et al. [7] have analyzed the dynamic characteristics of a wide inclined plane slider bearing. It is found that higher dynamic stiffness and damping coefficients are predicted for the bearing with small values of profile parameter. To provide more information for bearing designing, we are motivated to investigate the dynamic characteristics of the bearing with different film shapes.

On the basis of the thin-film lubrication theory, this study is mainly concerned with the dynamic characteristics of wide slider bearings with an exponential film shape including the effects of squeezing action. By applying a small perturbation technique to the dynamic Reynolds-type equation, both of the steady-state Reynolds equation and the perturbed-type Reynolds equation are derived. The steady-state performance and the dynamic characteristics of bearings at different inlet-outlet film ratios are then evaluated. Comparing with those of the plane sliders, characteristics of bearings are presented in terms of the steady load-carrying capacity, dynamic stiffness and damping coefficients.

\section{FORMULATION}

Figure 1 shows the geometrical configuration of a wide exponential shaped slider bearing including the effect of squeezing action. It is assumed that the fluid inertia is small, the side leakage is negligible, and the 
flow is incompressible and laminar. Based upon the thin-film theory of hydrodynamic lubrication, the dynamic Reynolds-type equation taking into account the squeezing action is derived by Lin et al. [7].

$$
\frac{\partial}{\partial x}\left(h^{3} \frac{\partial p}{\partial x}\right)=6 \mu U \frac{\partial h}{\partial x}+12 \mu V
$$

In the equation $p$ represents the film pressure, $\mu$ denotes the lubricant viscosity, $U$ is the sliding velocity of the lower surface, $V=\partial h / \partial t$ denotes the squeezing velocity of the upper surface, $h(x, t)$ is the film thickness depending upon the time $t$ and the coordinate $x$. For an exponential shaped slider, the film thickness $h(x, t)$ is described by:

$$
h(x, t)=h_{m}(t) \cdot \exp \left(-\frac{x}{L} \ln r\right)
$$

In the equation $h_{m}(t)$ denotes the minimum film thickness depending upon the time $t$ only, $L$ is the length of the upper pad, and the non-dimensional parameter represents the inlet-outlet film ratio defined by: $r=h_{1} /$ $h_{m}$. Introduce the non-dimensional variables and parameters defined by the following.

$$
x^{*}=\frac{x}{L}, \tau=\frac{U t}{L}, h_{m}^{*}=\frac{h_{m}}{h_{m 0}}, p^{*}=\frac{p h_{m 0}^{2}}{\mu U L}
$$

where $h_{m 0}(t)$ represents the minimum film height of the bearing operating under steady state. The non-dimensional dynamic Reynolds-type equation and the film thickness are then obtained.

$$
\begin{aligned}
& \frac{\partial}{\partial x^{*}}\left[h^{* 3}\left(x^{*}, \tau\right) \frac{\partial p^{*}}{\partial x^{*}}\right]=6 \frac{\partial h^{*}}{\partial x^{*}}+12 \frac{\partial h^{*}}{\partial \tau} \\
& h^{*}\left(x^{*}, \tau\right)=h_{m}^{*}(\tau) \cdot \exp \left(-x^{*} \ln r\right)
\end{aligned}
$$

Following a small perturbation technique by Ghosh

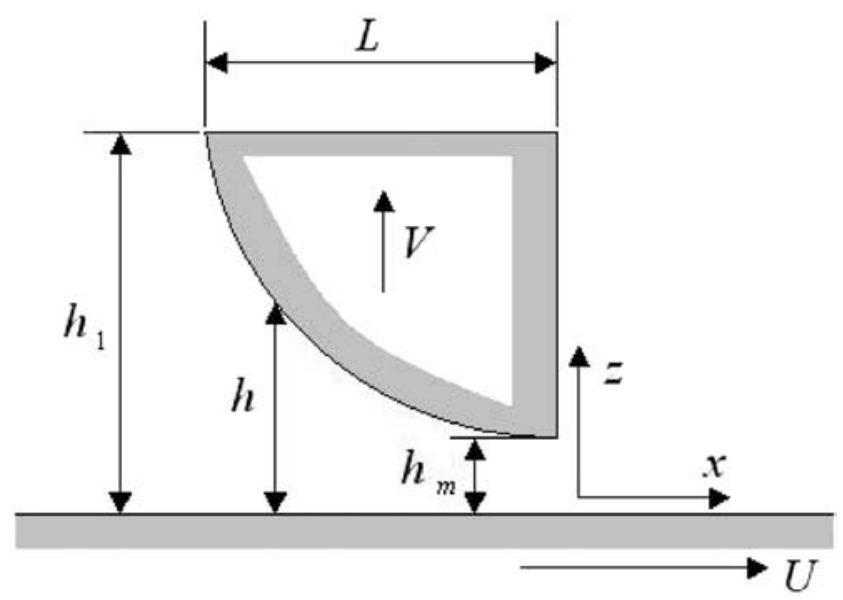

Fig. 1. Geometrical configuration of a wide exponential shaped slider bearing. and Majumdar [2] and Guha et al. [3], the bearing is assumed to undergo small-amplitude oscillations about its steady-state position. The film pressure and the film thickness are expressed as follows:

$$
p^{*}=p_{0}^{*}+p_{1}^{*} \varepsilon e^{i \tau}, h_{m}^{*}=1+\varepsilon e^{i \tau}
$$

where $\varepsilon$ represents a small amplitude of the oscillation. Substituting the above expressions into the non-dimensional dynamic Reynolds-type equation and neglecting higher-order terms of $\varepsilon$, one can obtains two Reynoldstype equations responsible for the steady-state performance and the dynamic stiffness and damping characteristics, respectively.

$$
\begin{aligned}
& \frac{\partial}{\partial x^{*}}\left[\exp \left(-3 x^{*} \ln r\right) \frac{\partial p_{0}^{*}}{\partial x^{*}}\right]=-6 \ln r \exp \left(-x^{*} \ln r\right) \\
& \frac{\partial}{\partial x^{*}}\left[\exp \left(-3 x^{*} \ln r\right) \frac{\partial p_{1}^{*}}{\partial x^{*}}\right]=i 12 \exp \left(-x^{*} \ln r\right) \\
& -6 \ln r \exp \left(-x^{*} \ln r\right)-3 \frac{\partial}{\partial x^{*}}\left[\exp \left(-3 x^{*} \ln r\right) \frac{\partial p_{0}^{*}}{\partial x^{*}}\right]
\end{aligned}
$$

Using the expression of equation (10), equation (11a) is written as:

$$
\begin{aligned}
& \frac{\partial}{\partial x^{*}}\left[\exp \left(-3 x^{*} \ln r\right) \frac{\partial p_{1}^{*}}{\partial x^{*}}\right]=i 12 \exp \left(-x^{*} \ln r\right) \\
& +12 \ln r \exp \left(-x^{*} \ln r\right)
\end{aligned}
$$

\section{STEADY-STATE PERFORMANCE}

If we neglect the cavitation effects at the ends, the steady-state film pressure can be found from the steady Reynolds-type equation (7) with the boundary conditions:

$$
p_{0}^{*}=0 \text { at } x^{*}=-1 \text { and } x^{*}=0
$$

The steady-state film pressure is then obtained.

$$
p_{0}^{*}=\frac{3}{\ln r} \exp \left(2 x^{*} \ln r\right)+c_{1} \frac{1}{3 \ln r} \exp \left(3 x^{*} \ln r\right)+c_{2}
$$

where $c_{1}$ and $c_{2}$ are found to be: ,

$$
c_{1}=\frac{-9[1-\exp (-2 \ln r)]}{1-\exp (-3 \ln r)},
$$




$$
c_{2}=\frac{-3}{\ln r}+\frac{3[1-\exp (-2 \ln r)]}{\ln r[1-\exp (-3 \ln r)]}
$$

The steady-state load-carrying capacity per unit width is evaluated by integrating the steady-state film pressure.

$$
W_{0}^{*}=\frac{W_{0} h_{m 0}^{2}}{\mu U L^{2}}=\int_{x=-1}^{0} p_{0}^{*} d x^{*}
$$

After performing the integration, one can obtains

$$
W_{0}^{*}=\frac{3[1-\exp (-2 \ln r)]}{2(\ln r)^{*}}+c_{1} \frac{1-\exp (-3 \ln r)}{9(\ln r)^{*}}+c_{2}
$$

\section{DYNAMIC STIFFNESS AND DAMPING CHARACTERISTICS}

If we neglect the cavitation effects at the ends, the perturbed film pressure is calculated from the perturbed Reynolds-type equation ( $8 b$ ) with the boundary conditions:

$$
p_{1}^{*}=0 \text { at } x^{*}=-1 \text { and } x^{*}=0
$$

The perturbed film pressure is then obtained.

$$
\begin{aligned}
p_{1}^{*} & =i \frac{-6}{(\ln r)^{2}} \exp \left(2 x^{*} \ln r\right)+\frac{-6}{\ln r} \exp \left(2 x^{*} \ln r\right) \\
& +c_{3} \frac{1}{3 \ln r} \exp \left(3 x^{*} \ln r\right)+c_{4}
\end{aligned}
$$

In the equation $c_{3}=c_{3 r}+i c_{3 i}$ and $c_{4}=c_{4 r}+i c_{4 i}$ are given by:

$$
\begin{aligned}
& c_{3 r}=\frac{18[1-x p(-2 \ln r)]}{1-\exp (-3 \ln r)}, \\
& c_{3 i}=\frac{18[1-\exp (-2 \ln r)]}{(\ln r)[1-\exp (-3 \ln r)]} \\
& c_{4 r}=\frac{18-c_{3 r}}{3 \ln r}, c_{4 i}=\frac{18-c_{3 r} \ln r}{3(\ln r)^{2}}
\end{aligned}
$$

The perturbed load-carrying capacity per unit width is evaluated by integrating the perturbed film pressure.

$$
W_{1}^{*}=\frac{W_{1} h_{m 0}^{2}}{\mu U L^{2}}=\int_{x^{*}=0}^{1} p_{1}^{*} d x^{*}
$$

Substituting the expression of $p_{1}^{*}$ into the above equation, one has

$$
W_{1}^{*}=\operatorname{Re} a l\left(W_{1}^{*}\right)+i \operatorname{Im} a g\left(W_{1}^{*}\right)
$$

$$
\begin{aligned}
\operatorname{Re} a l\left(W_{1}^{*}\right) & =c_{4 r}+c_{3 r} \frac{1-\exp (-3 \ln r)}{9(\ln r)^{2}} \\
& +\frac{-3[1-\exp (-2 \ln r)]}{(\ln r)^{2}} \\
\operatorname{Im} \operatorname{ag}\left(W_{1}^{*}\right) & =c_{4 i}+c_{3 i} \frac{1-\exp (-3 \ln r)}{9(\ln r)^{2}} \\
& +\frac{-3[1-\exp (-2 \ln r)]}{(\ln r)^{3}}
\end{aligned}
$$

The perturbed load-carrying capacity resulting from the perturbed film pressure can be written in terms of linear spring and damping coefficients.

$$
W_{1}^{*}=-K^{*}-i D^{*}
$$

Comparing the real part and imaginary part of equations (19) and (22), the dynamic stiffness coefficient and the dynamic damping coefficient are given respectively by:

$$
\begin{aligned}
& K^{*}=-\operatorname{Re} \operatorname{al}\left(W_{1}^{*}\right) \\
& D^{*}=-\operatorname{Im} \operatorname{ag}\left(W_{1}^{*}\right)
\end{aligned}
$$

\section{RESULTS AND DISCUSSION}

Taking into account the transient squeezing-action effects, the dynamic characteristics of a wide exponential shaped slider bearing are investigated. By applying a small perturbation technique, both of the steady-state performance and the dynamic characteristics are analyzed. In the present study, results of the bearing characteristics are presented with inlet-outlet film ratio $r=1.2-3.8$.

Figure 2 shows the variation of dimensionless steady load-carrying capacity $W_{0}^{*}$ with inlet-outlet film ratio $r$. The dashed curve shows the results for an inclined plane slider bearing obtained by Lin et al. [7]. The solid curve presents the steady load-carrying capacity for the present study. It is observed for both types of bearings that there exists a critical value of inletoutlet film ratio such that the value of steady loadcarrying capacity achieves the maximum. Comparing with those of the inclined plane slider, the exponential shaped slider provides a higher load-carrying capacity for larger values of the inlet-outlet film ratio.

Figure 3 presents the variation of dimensionless dynamic stiffness coefficient $K^{*}$ with inlet-outlet film ratio $r$. It is observed for the inclined plane slider that the maximum stiffness coefficient lies within the range of small values of the inlet-outlet film ratio. But the maximum stiffness shifts to the position of a larger $r$ for 


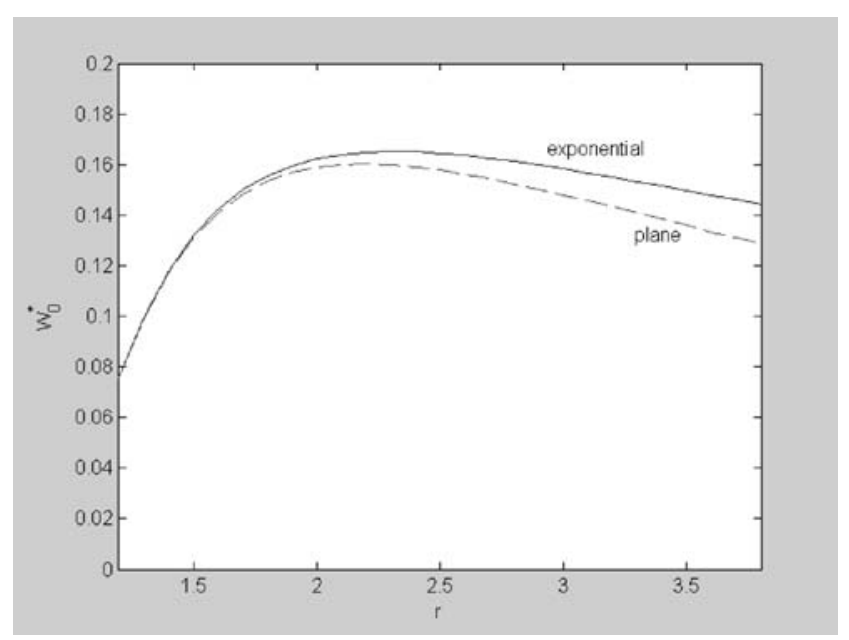

Fig. 2. Variation of dimensionless steady load-carrying capacity $W_{0}^{*}$ with inlet-outlet film ratio $r$.

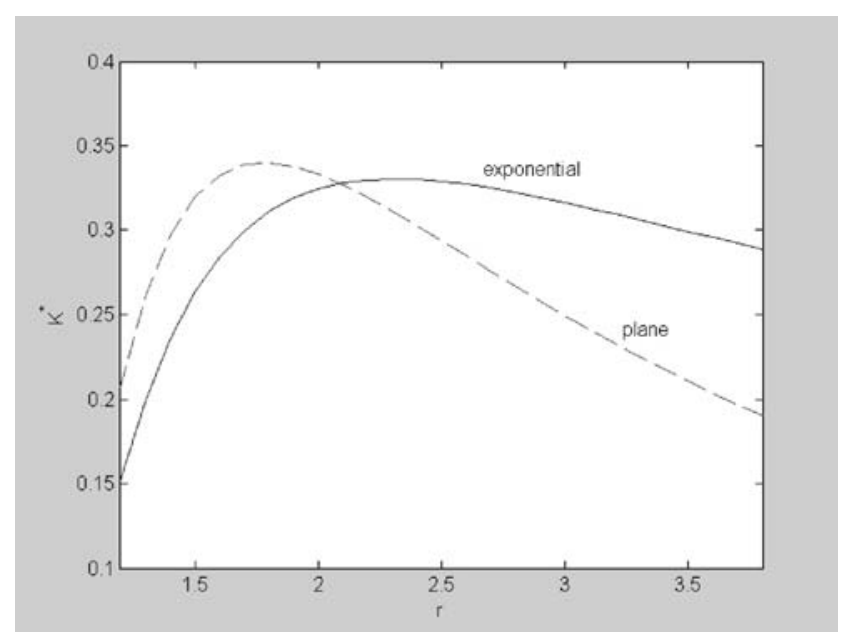

Fig. 3. Variation of dimensionless dynamic stiffness coefficient $K^{*}$ with inlet-outlet film ratio $r$.

the exponential shaped bearing. Comparing with the inclined-plane slider, the slider with an exponential shaped film results in a significantly increased stiffness for larger values of the inlet-outlet film ratio. Figure 4 shows the variation of dimensionless dynamic damping coefficient $D^{*}$ with inlet-outlet film ratio $r$. For both types of bearings, increasing value of $r$ yields a decreasing damping coefficient. Comparing with those of the inclined plane slider, the exponential shaped slider provides a higher damping coefficient, especially for larger values of the film ratio. Totally, larger values of the inlet-outlet film ratio for the exponential shaped slider provide higher load-carrying capacity and better dynamic stiffness and damping characteristics as com-

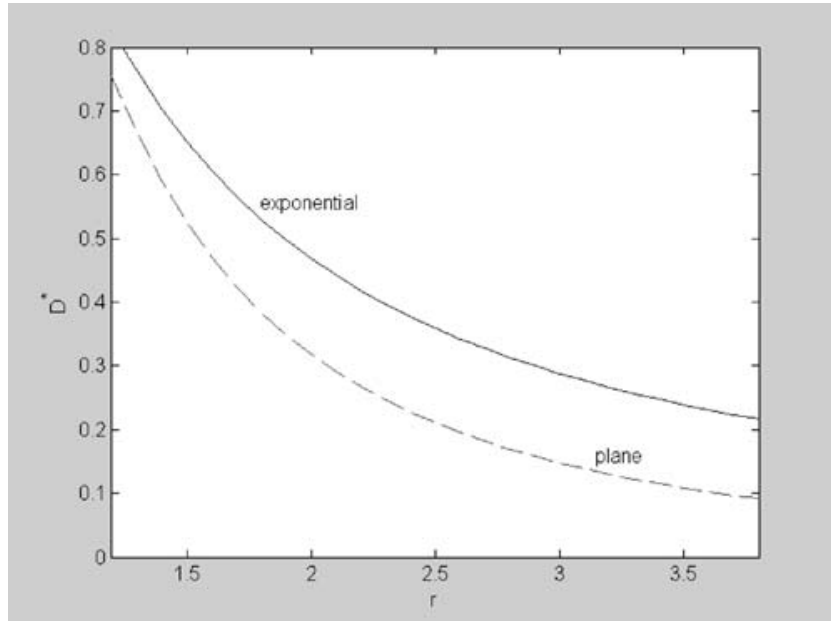

Fig. 4. Variation of dimensionless dynamic damping coefficient $D^{*}$ with inlet-outlet film ratio $r$.

pared to those of the inclined plane slider bearing.

\section{CONCLUSIONS}

On the basis of the thin-film lubrication theory, a study of dynamic characteristics for a wide exponential shaped slider bearing taking into account the squeezingaction effects is presented. By applying a small perturbation technique to the dynamic Reynolds-type equation, both the steady-state performance and the dynamic characteristics are calculated. According to the results discussed, conclusions can be drawn as follows.

Both of the steady-state performance and the dynamic characteristics are significantly affected by the inlet-outlet film ratio of slider bearings. There exists a critical film ratio such that the bearing possesses a maximum load-carrying capacity. Comparing with those of the inclined plane slider by Lin et al. [7], the exponential shaped slider provides higher load-carrying capacity and better dynamic stiffness and damping characteristics at larger values of the inlet-outlet film ratio. The finding of the present study provides useful information for engineers in designing and application of bearing systems.

\section{REFERENCES}

1. Chowdhury, S.J. and Ahmadi, G., "Thermodynamic Analysis of Wide Thrust Bearings Operating in Laminar Inertial Flow Regimes," ASME J. Lubric. Technol., Vol. 19, pp. 281-288 (1986).

2. Ghosh, M.K. and Majumdar, B.C., "Dynamic Stiffness and Damping Characteristics of Compensated Hydrostatic Thrust Bearings," ASME J. Lubric. Technol., Vol. 
104, pp. 491-496 (1982).

3. Guha, S.K., Ghosh, M.K., and Majumdar, B.C., "Rotordynamic Coefficients of Multirecess Hybrid Bearings Part II: Fluid Inertia Effect," Wear, Vol. 129, pp. 261-272 (1989).

4. Hamrock, B.J., Fundamentals of Fluid Film Lubrication, McGraw-Hill Inc., New York (1994).

5. King, K.F. and Taylor, C.M., "An Estimation of the Effect Fluid Inertia on the Performance of the Plane Inclined Slider Thrust Bearing with Particular Regard to Turbulent Lubrication," ASME J. Lubric. Technol., Vol. 99, pp. 129-135 (1997).

6. Launder, B.E. and Leschziner, M.L., "Flow in FiniteWidth, Thrust Bearings Including Inertia Effects," ASME J. Lubric. Technol., Vol. 100, pp. 330-338 (1987).

7. Lin, J.R., Lu, R.F., and Yang, C.B., "Linear Stability Analysis of a Wide Inclined Plane Slider Bearing," J. Marine Sci. Technol., Vol. 10, pp. 349-354 (2001).

8. Pinkus, O. and Sternlicht, B., Theory of Hydrodynamic Lubrication, McGraw-Hill Inc., New York (1961).

9. Qvale, E.B. and Wiltshire, F.R., "The Performance of Hydrodynamic Lubricating Films with Viscosity Variations Perpendicular to the Direction of Motion," ASME
J. Lubric. Technol., Vol. 94, pp. 44-48 (1972).

10. Rodkiewicz, C.M. and Anwar, M.I., "Inertia and Convective Effect in Hydrodynamic Lubrication of a Slider Bearing," ASME J. Lubric. Technol., Vol. 93, pp. 313-315 (1971).

11. Rodkiewicz, C.M., Hinds, J.C., and Dayson, C., "Inertia, Convective, and Dissipation Effects in the Thermally Boosted Oil Lubricated Sliding Thrust Bearing," ASME J. Lubric. Technol., Vol. 97, pp. 121-129 (1975).

12. Safar, Z.S., "Thermal and Inertia Effects in TurbulentFlow Thrust Bearing," Proc. I. Mech. E., Vol. 22, pp. 4345 (1980).

13. Synder, W.T., "The Nonlinear Hydrodynamic Slider Bearing," ASME J. Basic Engin., Vol. 85, pp. 429-434 (1963).

14. Talmage, G. and Carpino, M., "A Pseudospectral-Finite Difference Analysis of an Infinitely Wide Slider Bearing with Thermal and Inertia Effects," STLE Tribol. Transact., Vol. 40, pp. 251-258 (1997).

15. Taylor, C.M. and Dowson, D., "Turbulent Lubrication Theory-Application to Design," ASME J. Lubric. Technol., Vol. 96, pp. 36-46 (1974). 